\title{
Computational Personality Assessment
}

\author{
Clemens Stachl ${ }^{1}$ (D) , Ryan L. Boyd ${ }^{2,3,4}$ (D) , Kai T. Horstmann ${ }^{5}$ (D) , Poruz Khambatta ${ }^{6}$ (D) , \\ Sandra C. Matz ${ }^{7}$ (D) , Gabriella M. Harari ${ }^{1}$
}

[1] Department of Communication, Stanford University, Stanford, CA, USA. [2] Department of Psychology, Lancaster University, Lancaster, United Kingdom. [3] Security Lancaster, Lancaster University, Lancaster, United Kingdom. [4] Data Science Institute, Lancaster University, Lancaster, United Kingdom. [5] Institute of Psychology, HumboldtUniversity of Berlin, Berlin, Germany. [6] Anderson School of Management, UCLA, Los Angeles, CA, USA. [7] Columbia Business School, Columbia University, New York City, NY, USA.

Personality Science, 2021, Vol. 2, Article e6115, https://doi.org/10.5964/ps.6115

Received: 2021-02-08 • Accepted: 2021-06-21 • Published (VoR): 2021-07-15

Handling Editor: John F. Rauthmann, Bielefeld University, Bielefeld, Germany

Reviewing: Round 1 - Anonymous \#1; Anonymous \#2; Anonymous \#3. Open reviews are available [see Index of Supplementary Materials]

Corresponding Author: Clemens Stachl, Department of Communication, Building 120, Room 110, 450 Jane Stanford Way, Stanford University, Stanford, CA 94305, USA. E-mail: cstachl@stanford.edu

\section{Abstract}

Computational methods have increased the objectivity of measures of human behavior and positioned personality science to benefit from the ongoing digital revolution. In this review, we define and discuss computational personality assessment (CPA), a measurement process that uses computational technologies to obtain estimates of personality. We briefly review some of the most promising sources of data currently used for CPA: mobile sensing, digital footprints from social media, images, language, and experience sampling. We present a concise overview of key findings, discuss the promise and opportunities of CPA (e.g., moving towards objective measures of personality, obtaining new insights from big data), and highlight important limitations and challenges in the development and application of CPA (e.g., establishing reliability and validity, selecting appropriate ground truth criterion, assessing affect and cognition, implications for ethics and privacy). We conclude with our perspective on how CPA could change our understanding of individual differences.

\section{Keywords}

computational social science, personality, behavior, machine learning, psychological assessment 


\section{Relevance Statement}

Computational personality assessment based on digital footprints and high frequent behavioral data from in-vivo sensing studies could drastically change the concept of personality and its assessment.

\section{Key Insights}

- Computational personality assessment will drastically impact personality science.

- Objective measures of behavior, thought, and feelings will be essential.

- Summary of key data sources, results, opportunities, and challenges.

It has been said that the quality of a scientific discipline flows from its tools of measurement (Tal, 2020). Measurement in science is subject to constant improvement, allowing for the evermore precise quantification of phenomena which, in turn, leads to progress in both theoretical and practical realms. One of the most profound changes that has broadly affected psychological science is the ongoing shift towards computational measurement and assessment.

In personality psychology, the phenomena of interest are individuals and their characteristic patterns of thinking, feeling, and behaving (Funder, 2009). To measure personality, psychologists rely on various forms of assessment, including self-reports, objective personality tests (Ortner \& Schmitt, 2014), and behavioral observation. Such personality assessments are used to describe and evaluate individuals, compare them to others, and make predictions about how they might behave, think, and feel in various situations (Back et al., 2009; Ozer \& Benet-Martínez, 2006).

A core challenge for personality assessment is that the phenomena of interest are complex patterns in individual differences that partially manifest over time. These patterns are typically represented as latent constructs (e.g., traits and states, values, goals, identity) that are mostly assessed using self-report methods (Paulhus \& Vazire, 2007). However, it has repeatedly been highlighted that more objective data on naturalistic behavior is needed to improve measurements in psychological science (Baumeister et al., 2007; Furr, 2009). For example, a personality assessment goal might include determining the extent to which a person is sociable over time, in general (trait assessment), or in a given situation during a short period of time (state assessment). In order to measure a person's sociability at the trait or state level, they could be asked about their communication behavior using self-report methods or be observed engaging in communication behavior using naturalistic observation methods.

Over the past decade, advances in computing technologies for data collection (e.g., social media platforms, smartphones) and analysis (e.g., machine learning) have made behavioral observation studies increasingly viable (and valuable), enabling more and better use of objective measurements in addition to subjective questionnaire items (Yarkoni, 
2012). For example, consider that many everyday thoughts, feelings, and behaviors are now mediated, reflected, and often recorded by the ubiquitous apps, social media platforms, and other technologies that people use on a daily basis. These digital records can be collected, aggregated, and analyzed for business applications and research alike. By providing a means of observing personality-relevant information in daily life, computing technologies introduce new opportunities for the quantification of individual differences from observational data. The use of computational technologies for assessment can also help us overcome our own human limitations (e.g., memory limitations when providing self-reports) and biases in theory development (e.g., inability to consider the influence of many factors at the same time, innate tendency to converge to simple theories; Jolly \& Chang, 2019).

Research at the intersection of personality psychology and computer science has already begun to 1) harness computational technologies to collect and store new sources of personality-relevant data, and 2) model such data to describe, understand, and predict individual differences. Given that computational technologies have and will continue to change measurement in psychological science, here we provide an overview of the most promising sources of data and current approaches being used in computational personality assessment.

We define computational personality assessment (CPA) as a measurement process that uses computational technologies to obtain estimates of personality. Specifically, CPA uses digital indicators and computing technologies for data processing and analysis to identify patterns in personality-relevant data (e.g., phone logs, digital footprints) to estimate personality at various levels (e.g., traits, states, processes). In Figure 1 we provide a schematic overview of how computational personality assessment might capture the Big Five model at the trait and state levels.

In this paper, we focus on five sources of data that are essential to CPA: 1) mobile sensing, 2) digital footprints from social media platforms, 3) images, 4) language, and 5) experience sampling. The data from these sources are not completely independent from one another, but we find the different categories provide a useful way of organizing a state-of-the-art review of the research in this burgeoning area. We then highlight some core opportunities for research on personality assessment through a computational lens, and critically discuss the limitations and challenges that need to be addressed for CPAs to become standardized psychometric tools. Finally, we conclude with our perspective on how CPA could fundamentally change our understanding of individual differences in thoughts, feelings, and behaviors. 


\section{Sources of Data for Computational Personality Assessment}

Figure 1

Schematic Illustration of CPA

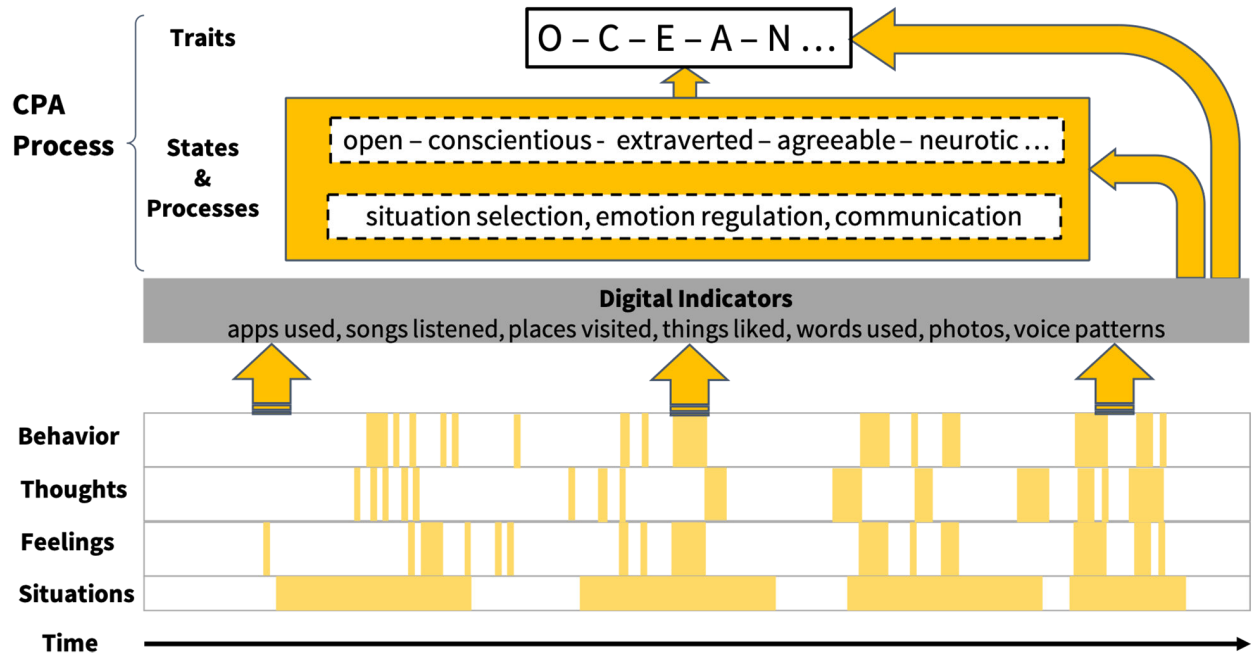

Note. Patterns of thoughts, feelings and behaviors across situations become accessible as digital indicators of personality through various sources of data. These indicators can either be used to infer personality traits directly or indirectly, via intermediary state or process prediction. Note that state and process assessments are listed as optional because computational trait assessment could either involve this intermediate step (e.g., recognizing momentary sociability) or could be performed on digital indicators directly (e.g., estimating extraversion trait levels from communication frequencies).

\section{Mobile Sensing}

Consumer electronics that are equipped with mobile sensing technologies (e.g., wearables, smartphones, IoT) are a pervasive feature of daily life for many people around the world. While as of 2017 , an estimated $5 \%$ people in developed nations ( $11 \%$ in developing) own smartwatches that they wear on their wrists, an estimated median of $91 \%(80 \%$ developing) of the developed world's population owns smartphones that they carry around as they go about their day, and an estimated $3 \%$ of people living in developed (6-10\% in developing) countries own smart home devices that reside in their homes ${ }^{1}$.

These technologies rely on mobile sensors (e.g., accelerometer, microphone, GPS) and metadata (e.g., call and SMS logs, app use logs) to provide services central to the func-

1) https://www2.deloitte.com/content/dam/Deloitte/us/Documents/technology-media-telecommunications/us-global-mobile-consumer-survey-second-edition.pdf 
tioning of the device, such as activity recognition, voice detection, information retrieval, or location tracking (Miller, 2012; Raento et al., 2009). Furthermore, these internet-connected devices are designed to exchange information for a more convenient user experience (e.g., a smartwatch syncing data to a mobile app). However, the data generated from these technologies can also be harnessed to obtain insights into personality-relevant information about their owners' behaviors (e.g., physical activity, social behavior, digital media use; Harari et al., 2017) and surrounding environments (e.g., location, ambience; Harari et al., 2018). As such, data from sensing technologies present several opportunities for those interested in assessing and understanding the manifestation of personality in daily life. In contrast to self-reports, sensing methodologies allow for the collection and quantification of objective data about people's thoughts, feelings, and behaviors.

To date, studies have primarily focused on demonstrating how self-reported personality trait levels can be estimated or inferred from sensing data, evaluating machine learning models that classify or predict a person's self-reported Big Five trait scores. Taken together, past work on the prediction of personality traits from smartphone data suggests that individual levels of personality traits can be predicted from sensing data above chance for most traits (e.g., Stachl, Au, et al., 2020). Some initial studies have also begun to examine the prediction of personality states from sensing data, suggesting predictability for Extraversion states only (Rüegger et al., 2020). Cumulatively, personality sensing research provides new insights into people's characteristic behavioral patterns (e.g., stable tendencies, dynamic variability) and new opportunities for automating personality assessment (e.g., self-reported or observed behavioral dimensions; Harari et al., 2020). In the future, we anticipate mobile sensing will usher in a new frontier in CPA within personality science.

\section{Digital Footprints From Social Media}

Social media (SM) has become an integral part of the lives of millions of people around the world (Bayer et al., 2020). Facebook alone has more than 2.6 billion active daily users; the WeChat platform is quickly catching up, with a reported 1.2 billion users in the first quarter of 2020. SM platforms - such as Facebook, Twitter, Instagram, Snapchat, or TikTok - facilitate a breathtaking diversity of human behavior including self-expression, communication, mobilization, transactions, and more. In doing so, they capture enormous amounts of data and information about their users: Their hopes and dreams, preferences and motivations, social connections, daily routines, and physical whereabouts can become reflected in the digital traces that people leave behind on these platforms (Matz \& Netzer, 2017).

The information captured via digital footprints from SM platforms includes both carefully curated identity claims as well as inadvertently generated behavioral residue (Gosling et al., 2011). For example, Facebook users can explicitly and deliberately communicate their interests and preferences using the "Like" button. In contrast, information 
about a user's current location, or the list of articles and ads they have clicked on while browsing web sites, reflects their preferences more implicitly. Early research into the psychological underpinnings of SM has shown that these seemingly innocuous traces can be used by human judges to make accurate inferences about the psychological traits of strangers, including their personality (Gosling et al., 2011). Although there is often an inherent tension between the desire to present oneself in an idealized way and the desire to present the "true" version of oneself, past research supports the proposition that users' SM profiles hold accurate and relevant information about their psychological traits (Back et al., 2010).

With the growing interest among psychologists in Big Data and machine learning, researchers have increasingly started to investigate the feasibility and validity of automated, computer-based predictions of personality from online digital footprints. For example, computational modeling has been used to successfully predict self-reported Big Five personality trait levels from Facebook Likes (Kosinski et al., 2013; Youyou et al., 2015), status updates (Park et al., 2015; Schwartz et al., 2013), and transaction data (Gladstone et al., 2019). Predictions of personality from SM profiles have not only been shown to be more accurate that the judgements made by colleagues, friends and family members (Youyou et al., 2015), but they are also known to predict external criteria equally well or even better than people's self-reported responses (Park et al., 2015).

The unprecedented ability to automatically retrieve information on peoples' personality at scale has paved the way for SM platforms and third parties to use insights into people's personality to shape their experience on the platforms themselves. For example, SM platforms and marketers can utilize predictions of personality traits from digital footprints to tailor the content a user is exposed within a platform to their specific psychological needs and preferences (Matz et al., 2017).

\section{Images}

Images, including those shared on SM platforms, personal and professional websites, or offline settings, are rich sources of information for personality assessment. Cameras are embedded in many everyday devices (e.g., smartphones, cars) and are used extensively in both private and public settings (e.g., security systems in homes, CCTV systems), providing further visual information about individuals and their behavior that may be recorded and analyzed automatically (e.g., Philpot et al., 2020).

Though the human brain is carefully attuned to make sense of the vast stream of visual data our eyes perceive, this has historically proven much harder for computers. Nonetheless, with the advent of modern computing resources and improved algorithms, researchers have made great strides in the field of computer vision. For example, in recent years, scientists have greatly increased our ability to computationally detect objects, track motion, recognize actions, and estimate poses from image data (Voulodimos et al., 2018). Thus, it is possible that the same technologies that have enabled recent 
breakthroughs may also allow researchers to analyze images for personality content and facilitate the automatic assessment of personality traits and states.

Early work in this space has shown promising results. In one study, participants were fitted with a wearable camera, which automatically took pictures of their environment throughout the day. Objects in the captured images were automatically classified via computer vision algorithms and were shown to predict both self-reported personality traits and situational characteristics (Blake et al., 2020). In related work, by analyzing online images collected from SM, researchers demonstrated that it is even possible to detect personality traits from combinations of low-level image features (e.g., color diversity, image size, number of faces; Segalin, Celli, et al., 2017).

Moreover, faces can reflect a person's emotional expressions and transient states, but could they offer clues to more enduring psychological dispositions? The face is one of the most central aspects of a person's image, yet it remains relatively underexplored as a personality-relevant feature of image data. Alongside the progress of the big data revolution, facial images and videos are becoming ever more prevalent, making appearances in profile pictures, SM posts, and online meetings. Even beyond the digital realm, faces are both easily accessible and extremely salient stimuli for human beings. Initial investigations in this area show some evidence that this may be possible. Researchers have demonstrated that machine learning models can be trained to predict psychological dispositions, including personality, from images, such as those on SM (Kachur et al., 2020).

Nonetheless, it is important to note some limitations present in research using images for personality assessment. First, it is unknown whether such predictions rely on natural facial appearance or other low-level image cues, such as ambient lighting in the photograph. For example, extroverts are more likely to take pictures in bright environments (Segalin, Perina, et al., 2017), and it may be these features, rather than those related to faces that a model is using to form its judgments. Second, no matter how advanced computer vision becomes, images and faces in particular may not perfectly reflect an individual's personality (Todorov et al., 2015), so it is unlikely image-based detection can fully supplant other forms of personality assessment in terms of their reliability and validity. Third, while static images may convey significant information, the richness and temporal qualities found in videos may allow for even more insight into personality. Researchers have already started to explore video data to generate automatic personality assessments (Suen et al., 2019).

\section{Language}

Language data (e.g., data captured from verbal behavior, broadly defined, such as written text or audio recordings) is a central type of personality-relevant information available via computing technologies, such as SM and smartphone apps. Moreover, following standardized self-report questionnaires, language data being used as a source of diag- 
nostic information has perhaps one of the oldest traditions in the field of personality assessment. Psychologists have privileged verbal behavior - particularly written and spoken words - as something of a direct pipeline to personality itself. Dating back even to before Psychology's formative days, a person's words were treated as a distillate of their underlying drives, emotions, and thought patterns: the swirling miasma of the preconscious, or the very essence of what ultimately makes someone who they are as a person (Allport, 1942).

Early on, language data within personality science often consisted of conspicuous responding to explicit prompts - such as dream reports, self-description tasks, or projective tests - or cultural artifacts, such as books, newspaper articles, and so on (e.g., Stone et al., 1966). For the past several decades, language data has also been captured as part of the broader stream comprising one's environment and their interactions with the environment, such as intermittent audio recordings in daily life (Mehl et al., 2001). The proliferation of smaller, more affordable, and more accessible computer technology and SM has made the collection and automated analysis of language data increasingly available to researchers across disciplines. Today, it is possible to scrape, quantify, and analyze years of an individual's life through the language in their SM posts in minutes, and recent advances in computer processing promises that audio data will soon be an area of increased interest.

Today, the theoretical underpinnings of analysis of language data are relatively simple when compared to those of the early-to-mid 20th century. From a modern psychometric perspective, language data is most often (though not always) treated as a reflection of one's attentional habits, loosely defined (e.g., Tausczik \& Pennebaker, 2010). Extraverts, for example, tend to pay more attention to their social environments and other people; consistent with this definition, research finds that extraverts tend to use more "social" words - that is, words about people (e.g., "friend", "sister", "colleague"), generally speaking (e.g., Brown et al., 2020). Importantly, there have been recent calls for expansion of theory and assessment of verbal behavior to more meaningfully align with broader psychological and social theory (Boyd \& Schwartz, 2021).

Language data has been used extensively for the passive, non-invasive assessment of personality across a variety of study paradigms. More specifically, personality research with verbal behavior commonly takes language data in its raw, "unstructured" form; words are coded as belonging to different domains: emotions (e.g., "happy", "sad", "nervous"), sociality ("friend", "family", "togetherness"), metacognitive ("think", "understand", "wonder"), and so on (e.g., Boyd et al., 2015). SM has become the most common starting point for CPA from language data, with most research centering around Facebook (e.g., Schwartz et al., 2013), and Twitter (Sterling et al., 2020) as data sources. While much of the contemporary language-based research on personality is driven by the goal of maximizing out-of-sample predictive performance - particularly inferences of the Big 
Five (e.g., Hall \& Caton, 2017) - others have used language data as the material by which personality itself is conceptualized (Kulkarni et al., 2018).

\section{Experience Sampling}

Experience sampling methodology (ESM; also known as Ecological Momentary Assessment [EMA]) describes the repeated assessment of a person's thoughts, feelings, behaviors through active self-reporting (Horstmann \& Ziegler, 2020). Several characteristics of EMA make it relevant for CPA: It can provide subjective labels to purely objective data (e.g., labelling stressful experiences) for the training of predictive models (e.g., recognizing personality states and traits; Judge et al., 2014). ESM does not only allow collecting self-reports on rating-scales, but more generally describes a method in which the participant intentionally provides information about their current thoughts, feelings, behaviors, but also their motives or environments (e.g., Brown et al., 2017). Hence, ESM can also provide self-reported audio or video data as an addition to conventional self-report items that can help to sample personality-relevant objective data (Buschek et al., 2018). Finally, newer theories of personality make predictions about dynamic within-person processes - that is, how a person behaves, thinks, or feels) from moment to moment in interaction with their current environment (e.g., Whole Trait Theory; Fleeson \& Jayawickreme, 2015).

Current ESM approaches that allow for a somewhat standardized (yet adaptive) computational interaction with participants - for example via artificial conversational agents (i.e., chatbots) - could allow for the investigation of more complex and dynamic aspects of personality (e.g., communication processes) and apply interventions (e.g,. personality change; Stieger et al., 2021). ESM is not a fully automated process in the sense that participants need to actively provide information, but the so-obtained data can still play a major role in CPA. Finally, ESM provides a relatively well-understood source of self-report data that can be used as a complementary reference point when evaluating new approaches to the assessment of personality.

\section{Discussion}

Having provided a review of research centered on the most promising types of data for $\mathrm{CPA}$, we next turn to a discussion of the key opportunities, challenges, and directions for this area. In doing so, our aim is to outline how these sources of data might catalyze new directions of personality research and assessment, while critically discussing the current limitations of these methods and how they might be addressed moving forward. 


\section{Opportunities}

Computational approaches to personality assessment provide new ways of quantifying individual differences in thoughts, feelings, and behavior and estimating those differences, in an increasingly potentially automated fashion. The field of personality psychology has begun to recognize the added value of CPA to traditional forms of personality assessment (e.g., cheaper, more accurate, different aspects of personality). The degree to which CPA continues to expand within the discipline will depend, in part, on its ability to help solve pressing real-world problems (e.g., behavior-change for climate action, recognize and prevent psychopathology, better matching of romantic partners and jobs with applicants). Perhaps the most promising aspects of CPA lie in 1) its increased reliance on more objective sources of data (in comparison with self-reported personality inventories) and 2) its ability to accurately model more complex and dynamic patterns of individual difference in large-scale datasets. Both aspects could help to improve the assessment and the conceptualization of personality at state, trait, and process levels.

\section{Towards Objective Measures of Personality}

In self-report personality questionnaires, people are asked about their typical behaviors, thoughts, and feelings (e.g., their tendency to socialize or be honest in relationships), which can trigger socially desirable response biases. Digital footprints and mobile sensing data in contrast could potentially allow for the automated, objective quantification of a person's behaviors, thoughts, and feelings (e.g., their tendency to communicate with others or use dating apps while in a relationship). In that sense, objective behavioral data for CPA could help to overcome the well-known problems of questionnaire-based assessments (e.g., memory, social desirability, response-styles, faking) and reduce participant burden by enabling passive and automated personality assessment. Moreover, objective behavioral data can be combined with self-reported assessments to provide complementary information on personality traits and states. Consider, for example, a person who spends little time communicating with others (based on sensed data) but reports being a talkative person - this discrepancy may itself reveal key personality information that would not otherwise be available through either source of data by itself.

\section{Insights From Big Data}

Behavioral observations from digital data sources can reflect personality-relevant information, but do not necessarily represent a person's standing on personality dimensions (e.g., sociability, honesty) on their own. However, when these and other indicators are considered in tandem using computational modeling, they can reveal behavioral patterns that are indicative of a person's standing on latent personality dimensions (e.g., outgoing phone calls at night and daily use of communication apps as indicators of Extraversion; Stachl, Au, et al., 2020). 
It is also known that some aspects of human personality are better assessed by the self, some are better assessed by others, and some aspects are neither assessed well by the self nor others (Vazire \& Mehl, 2008). For example, people are poor estimators of their online media use (Parry et al., 2021), and most people do not have a complete picture of other peoples' online activities. Hence, to assess such blind-spots in personality expression, objective CPAs are necessary.

CPA will enable the quantification of personality expressions in a more fine-grained and holistic way that better accounts for the dynamic components of personality. Specifically, will it allow us to simultaneously model behavioral, situational, cognitive, and emotional processes in a dynamic fashion over time, as described in contemporary personality theories (e.g., Whole Trait Theory; Fleeson \& Jayawickreme, 2015). This, in turn, will finally enable researchers in personality science to better describe, understand, and anticipate the effects of personality.

\section{Limitations and Challenges}

Despite these promising opportunities, there are several challenges that limit the ability to use these new technologies for personality assessment. One frequent argument against using digital footprints, mobile sensing, and other digital sources of data for assessment purposes is that these new techniques still lack basic evaluations of their psychometric properties (e.g., reliability, validity). For CPAs to be a viable complementary approach to more traditional self-report assessments in applied settings, these new assessments must demonstrate adequate reliability (e.g., over time) and validity (e.g., by predicting relevant life outcomes). For example, a starting point could be to evaluate the extent to which the new assessment's properties compare to the lowest thresholds for reliable and valid self-reported personality assessments (e.g., single-item trait measures).

\section{Establishing Reliability}

CPAs from digital behavioral data are also impaired by the lack of understanding in how reliable obtained personality inferences might be. Several phenomena that are particularly relevant to these new approaches must be considered. For example, concept drifts (cf. longitudinal measurement invariance in psychometrics) refer to a change in the informativeness of digital behavioral indicators that are used as predictors with regard to a criterion (Lu et al., 2019). This effect can lead to the deterioration of a computational model for personality assessment over time. In addition, measurement inaccuracies that are induced by the methods themselves can lead to differences in observations. For example, smartphone sensors across device manufacturers may have different sampling rates (Geyer et al., 2020), data for assessments might not be available for everybody (e.g., digital footprints on social media), and the input data itself is subject to substantial variation across measurements (e.g., facial images across situations; Jenkins et al., 2011). Finally, the use of CPA models may not be readily extended to populations from 
which training data was not sampled (e.g., "liking" Poutine on Facebook in Canada may be differentially indicative of a person's openness levels than liking it in India). The development of reliable CPAs will require more interdisciplinary work across several disciplines, within and across institutions (e.g., Psychology, Computer Science, Philosophy and Ethics, industry, academia, and the general public). Moreover, to make personality science truly computational, the education of future researchers at universities (e.g., $\mathrm{PhD}$ students) will need to be adapted to include more computational (e.g., programming), ethical (e.g., open science, privacy), and mathematical modules (e.g., machine learning, measurement theory).

\section{Validating Assessments}

Another big challenge in CPA is to test the validity of newly developed models (Tay et al., 2020). In particular, questions arise with regard to whether traditional conceptualizations of validity can be applied "as is" to CPAs (Bleidorn \& Hopwood, 2019; Stachl, Pargent, et al., 2020). For example, traditional conceptualizations used with self-report data, such as the retainment of maximally linear convergent items in a factor structure, may not apply to computational methods because effects might be non-linear and this criterion may not necessarily lead to higher predictive performances, which are the standard by which most predictive computational methods are evaluated (Tay et al., 2020). Moreover, digital behavioral data may be indicative of multiple personality dimensions simultaneously, which is inherently different from how self-report measures are developed (e.g., items tend to be used to derive a personality score for a single dimension).

For example, the item "I am a talkative person" is used solely as an indicator of Extraversion in personality trait inventories (unidimensional), but the average number of calls a person makes at night could be an indicator for levels of sociability and self-consciousness (e.g., Stachl, Au, et al., 2020). Such unresolved issues complicate the validation and development of these new approaches and will need to be overcome to transform CPA research from pure prediction to standardized psychometric tools. One potential way to evaluate the validity of CPA is to map out the nomological network with regard to relevant life outcomes (e.g., health, occupation satisfaction, and performance; Bleidorn \& Hopwood, 2019). For example, the findings from a study using Facebook Likes to predict Big Five traits showed that the computational assessments were associated with life outcomes (e.g., substance use), suggesting some evidence of external predictive validity (Youyou et al., 2015). However, more specific work is needed with regard to how computational assessments should correlate with life outcomes and at which magnitude. For example, personality assessments are typically used for partner matching and recruiting, so behavior-based CPAs could for some time be compared with traditional forms of assessment (i.e., standardized self-reports) in their ability to predict outcomes in these domains (e.g., relationship satisfaction, job performance). 


\section{The Ground Truth Challenge}

Central to the challenge of establishing validity is a more foundational issue regarding the appropriate "ground truth" criteria for evaluating CPAs. The ground truth problem can be boiled down to a conceptual question: What is the most representative and unbiased conceptualization of systematic patterns in human feelings, thoughts, and behavior (i.e., personality)?

Virtually all of the latest computational approaches to personality assessment rely on self-reported personality trait scores as the ground truth criteria for evaluating the performance of the newly derived assessment models (e.g., Gladstone et al., 2019; Stachl, Au, et al., 2020; Youyou et al., 2015). This circumstance is challenging, because it means that computational assessments are also somewhat limited by the methodological biases and issues that are inherent to questionnaire-based measures - the outcomes these models typically aim to predict (Boyd \& Pennebaker, 2017). Moreover, the direct reliance on self-reports during the development and evaluation of computational models makes it difficult to argue for advantages of computational approaches in comparison to questionnaire-based methods. For these kinds of models, it is necessary to have "labeled" data for models to be trained on, but by no means does that mean that self-reports are the ultimate criterion to compare against or that models should be optimized to predict. On the contrary, we argue that it should be apparent that aggregated self-reports of peoples' behavioral tendencies are naturally inferior to direct behavioral assessments of those same behavioral patterns.

However, it is questionable how useful the concept of ground truth really is in CPA in general. It might be rather useful to think of self- and other reported personality information to be complementary to purely behavioral measures (Vazire \& Mehl, 2008). While this is no easy problem to solve, one way forward could be to explore whether the full breadth of new data sources can be statistically combined to allow for the identification of a comprehensive taxonomy of individual differences that predict relevant life outcomes and future personality states with reasonable accuracy. By doing so, CPA could also play a major role in the further conceptualization of personality itself. For example, it is possible that computational models of distinct patterns in thoughts, feelings, and behaviors that are predictive of life outcomes could reflect different (e.g., narrower and more contextual) conceptualizations of individual differences (e.g., a good salesman) while also considering situational dependencies (Rauthmann \& Sherman, 2018).

\section{Affect and Cognition}

Putting issues of validity aside, the question of how to best conceptualize affective and cognitive components of personality in an objective manner remains unsolved. The inherent subjectivity of thoughts and feelings, make it very difficult to directly measure them in objective ways (Sen et al., 2019). Related to most extant research, our description of CPA in this manuscript has primarily focused on behavior. However, in 
order to realize a comprehensive assessment of personality, it is necessary yet much more challenging to observe and quantify what people think and feel.

Some recent studies hint at the possibility of measuring cognitive (Jach et al., 2020) and affective (e.g., Israel \& Schönbrodt, 2021) personality-related processes and states using computational modeling. These approaches are good starting points for further research and highlight the potential of digital physiological metrics (e.g., muscular and brain activity) for the assessment of personality traits and states. Computational modeling will likely play a decisive role in advancing research in this area. One example is the increased use of complex, context-aware transformer models to process human language data (e.g., Devlin et al., 2019). These models can provide digital representations of human language (e.g., text) while accounting for at least one level of context (Boyd \& Schwartz, 2021). Considering their success in natural language processing, it is not far-fetched to imagine the use of these techniques to create representations of other sequential, personality-relevant types of data (e.g., physiological, behavioral, and cognitive signals). Put another way - in the near future, we anticipate an increasing ability to not only measure behaviors, but to ensure that those measurements are modeled in a psychologically accurate way that accounts for context (e.g., fidgeting while talking to a romantic interest versus fidgeting while talking to a disliked coworker).

\section{Ethics and Privacy}

All research that has the potential to inform or influence people's lived experiences - particularly work related to people's personal thoughts, feelings and behaviors comes with inherent ethical challenges; CPAs are no exception. One of the most salient ethical implications of automated CPAs is the potential loss in agency and control of the assessed over the diagnostic process. In contrast to self-report questionnaires that are widely used for personality assessment, computational assessments do not require people to actively provide data for assessment. This is the case when the methods that are used for CPA, reveal patterns that would otherwise have remained hidden in the data (e.g., in faces; Kosinski, 2021). Hence, the use of facial images in psychometric assessments in particular has been subject to criticism due to the scalability of the method and the inability to avoid assessments (e.g., ratings through CCTV). It has been debated whether images taken on a public CCTV camera should ever be analyzed using such algorithms and acknowledgement of the highly problematic, pseudoscientific history of physiognomy ${ }^{2}$ - both in science and its gruesome real-world consequences (Gray, 2004).

Another ethically challenging aspect of CPAs is the potentially persistent accountability of people for their past actions that is created by the comprehensiveness of the data collection process involved and the online-persistence of digital footprints and created models (Slavkovik et al., 2021). For example, if computational personality inferences are

2) https://medium.com/@blaisea/physiognomys-new-clothes-f2d4b59fdd6a 
used to decide if a person will get a job, be recommended for promotion, is likely to graduate from college, or is a suitable partner to recommend on a dating app (as it is the case with self-reported personality), this can raise ethical questions for several reasons. First, if assessments are made without consent, or cannot be changed easily, this could result in a situation in which people must indefinitely fear the consequences of every single click or utterance, constraining people's freedom to express themselves free from judgment in an open society. Such concerns in turn could lead to intentional and persistent changes in peoples' behavior (e.g., behave as if you were extraverted) to avoid negative outcomes. These reactive, camouflaging behaviors in turn, could cause CPAs themselves to no longer be accurate. Moreover, the issue of accountability can aggravate when combined with a lack of algorithmic fairness and undetected model drifts (Kusner \& Loftus, 2020; Lu et al., 2019). In those cases, the accuracy of computational assessments could deteriorate over time and be systematically different for subgroups (e.g., women versus men). To improve this situation, a radical transparency in all steps of CPA will be necessary to detect, counteract, and prevent such issues.

Finally, it is possible that different conceptualizations of individual privacy and related ethical positions will influence the use of CPAs under different cultural and political norms. Whereas China has taken a more aggressive approach towards automated computational assessments and algorithmic scoring mechanisms ${ }^{3}$, the EU's recently published proposal for the regulation of AI suggests that the block will heavily regulate automated social scoring based on personality or social behavior ${ }^{4}$. It remains to be seen how legal frameworks will impact the development and the adoption of CPAs in different regions and areas of application. While the unregulated distribution of digital data has raised concerns that these practices will inevitably threaten individual privacy, clear ethical guidelines for CPA need to be developed (see, e.g., Lazer et al., 2020). Ever-evolving guidelines will need to consider how to balance the sensitivity of the data that is necessary to achieve most reliable and valid estimates in relation to the goal of the assessment with individual right on privacy.

\section{Conclusion}

Scientific progress is often characterized by improvements in the methods that it uses to investigate and conceptualize phenomena of interest. In personality science, we are on the path from personality prediction towards CPA, but remaining issues with regard to the objectivity, reliability, validity, and ethical aspects of CPAs must first be addressed. While CPA is still in its infancy, it is poised to both drastically change how we assess personality and how we think about it conceptually.

3) https://www.theatlantic.com/magazine/archive/2020/09/china-ai-surveillance/614197/

4) https://digital-strategy.ec.europa.eu/en/library/proposal-regulation-european-approach-artificial-intelligence 
Funding: This research was supported in part by a Stanford HAI Seed Grant, the National Science Foundation (SES-1758835), the Federal Bureau of Investigation (15F06718C0002523), and the Swiss National Science Foundation (196255).

Acknowledgments: We want to thank the reviewers, the scientific community, and the organizational behavior reading group at the University of Zurich, $\mathrm{CH}$ for their helpful input on an earlier version of the manuscript.

Competing Interests: Sandra Matz is a member of the editorial board of the journal.

Author Contributions: Clemens Stachl-Idea, conceptualization | Visualization (data presentation, figures, etc.) | Writing | Feedback, revisions | Project coordination, administration. Ryan L. Boyd-Visualization (data presentation, figures, etc.) | Writing | Feedback, revisions. Kai T. Horstmann-Writing | Feedback, revisions. Poruz KhambattaWriting | Feedback, revisions. Sandra C. Matz-Writing | Feedback, revisions. Gabriella M. Harari-Idea, conceptualization | Visualization (data presentation, figures, etc.) | Writing | Feedback, revisions | Supervision, mentoring | Funding to conduct the work.

Ethics Statement: No ethical issues and/or ethics approvals need to be disclosed.

Related Versions: Before submission of the manuscript to Personality Science, we had uploaded a pre-print to the Open Science Framework (OSF). The pre-print is openly accessible via https://psyarxiv.com/ck2bj/.

\section{Supplementary Materials}

For this article an Open Peer-Review is available via PsychArchives (for access see Index of Supplementary Materials below).

\section{Index of Supplementary Materials}

Personality Science. (Ed.). (2021). Supplementary materials to: Computational personality assessment [Open peer-review]. PsychOpen GOLD. https://doi.org/10.23668/psycharchives.4973

\section{References}

Allport, G. W. (1942). The use of personal documents in psychological science. Social Science Research Council Bulletin, 49.

Back, M. D., Schmukle, S. C., \& Egloff, B. (2009). Predicting actual behavior from the explicit and implicit self-concept of personality. Journal of Personality and Social Psychology, 97(3), 533-548. https://doi.org/10.1037/a0016229

Back, M. D., Stopfer, J. M., Vazire, S., Gaddis, S., Schmukle, S. C., Egloff, B., \& Gosling, S. D. (2010). Facebook profiles reflect actual personality, not self-idealization. Psychological Science, 21(3), 372-374. https://doi.org/10.1177/0956797609360756 
Baumeister, R. F., Vohs, K. D., \& Funder, D. C. (2007). Psychology as the science of self-reports and finger movements: Whatever happened to actual behavior? Perspectives on Psychological Science, 2(4), 396-403. https://doi.org/10.1111/j.1745-6916.2007.00051.x

Bayer, J. B., Triêu, P., \& Ellison, N. B. (2020). Social media elements, ecologies, and effects. Annual Review of Psychology, 71, 471-497. https://doi.org/10.1146/annurev-psych-010419-050944

Blake, A. B., Lee, D. I., De La Rosa, R., \& Sherman, R. A. (2020). Wearable cameras, machine vision, and big data analytics: Insights into people and the places they go. In S. E. Woo, L. Tay, \& R. W. Proctor (Eds.), Big data in psychological research (pp. 125-143). American Psychological Association. https://doi.org/10.1037/0000193-007

Bleidorn, W., \& Hopwood, C. J. (2019). Using machine learning to advance personality assessment and theory. Personality and Social Psychology Review, 23(2), 190-203. https://doi.org/10.1177/1088868318772990

Boyd, R. L., \& Pennebaker, J. W. (2017). Language-based personality: A new approach to personality in a digital world. Current Opinion in Behavioral Sciences, 18, 63-68. https://doi.org/10.1016/j.cobeha.2017.07.017

Boyd, R. L., \& Schwartz, H. A. (2021). Natural language analysis and the psychology of verbal behavior: The past, present, and future states of the field. Fournal of Language and Social Psychology, 40(1), 21-41. https://doi.org/10.1177/0261927X20967028

Boyd, R. L., Wilson, S. R., Pennebaker, J. W., Kosinski, M., Stillwell, D. J., \& Mihalcea, R. (2015). Values in words: Using language to evaluate and understand personal values. Proceedings of the 9th International Conference on Web and Social Media, ICWSM 2015, 31-40.

Brown, N. A., Blake, A. B., \& Sherman, R. A. (2017). A Snapshot of the Life as Lived. Social Psychological \& Personality Science, 8(5), 592-600. https://doi.org/10.1177/1948550617703170

Brown, T. B., Mann, B., Ryder, N., Subbiah, M., Kaplan, J., Dhariwal, P., Neelakantan, A., Shyam, P., Sastry, G., Askell, A., Agarwal, S., Herbert-Voss, A., Krueger, G., Henighan, T., Child, R., Ramesh, A., Ziegler, D. M., Wu, J., Winter, C., Hesse, C., Chen, M., Sigler, E., Litwin, M., Gray, S., Chess, B., Clark, J., Berner, C., McCandlish, S., Radford, A., Sutskever, I., \& Amodei, D. (2020). Language Models are Few-Shot Learners. arXiv. http://arxiv.org/abs/2005.14165

Buschek, D., Völkel, S., Stachl, C., Mecke, L., Prange, S., \& Pfeuffer, K. (2018). Experience sampling as information transmission: Perspective and implications. UbiComp '18: Proceedings of the 2018 ACM International foint Conference and 2018 International Symposium on Pervasive and Ubiquitous Computing and Wearable Computers, 606-611. https://doi.org/10.1145/3267305.3267543

Devlin, J., Chang, M.-W., Lee, K., \& Toutanova, K. (2019). BERT: Pre-training of deep bidirectional transformers for language understanding. Proceedings of the 2019 Conference of the North \{A\}merican Chapter of the Association for Computational Linguistics: Human Language Technologies (Long and Short Papers), 1, 4171-4186. https://doi.org/10.18653/v1/N19-1423

Fleeson, W., \& Jayawickreme, E. (2015). Whole Trait Theory. Fournal of Research in Personality, 56, 82-92. https://doi.org/10.1016/j.jrp.2014.10.009 
Funder, D. C. (2009). Persons, behaviors and situations: An agenda for personality psychology in the postwar era. Journal of Research in Personality, 43(2), 120-126. https://doi.org/10.1016/j.jrp.2008.12.041

Furr, R. M. (2009). Personality psychology as a truly behavioural science. European fournal of Personality, 23(5), 369-401. https://doi.org/10.1002/per.724

Geyer, K., Ellis, D. A., Shaw, H., \& Davidson, B. (2020). Open source smartphone app and tools for measuring, quantifying, and visualizing technology use. PsyArXiv. https://doi.org/10.31234/osf.io/eqhfa

Gladstone, J. J., Matz, S. C., \& Lemaire, A. (2019). Can psychological traits be inferred from spending? Evidence from transaction data. Psychological Science, 30(7), 1087-1096. https://doi.org/10.1177/0956797619849435

Gosling, S. D., Augustine, A. A., Vazire, S., Holtzman, N., \& Gaddis, S. (2011). Manifestations of personality in online social networks: Self-reported facebook-related behaviors and observable profile information. Cyberpsychology, Behavior, and Social Networking, 14(9), 483-488. https://doi.org/10.1089/cyber.2010.0087

Gray, R. T. (2004). About face: German physiognomic thought from Lavater to Auschwitz. Wayne State University Press.

Hall, M., \& Caton, S. (2017). Am I who I say I am? Unobtrusive self-representation and personality recognition on Facebook. PLOS ONE, 12(9), Article e0184417. https://doi.org/10.1371/journal.pone.0184417

Harari, G. M., Müller, S. R., Aung, M. S., \& Rentfrow, P. J. (2017). Smartphone sensing methods for studying behavior in everyday life. Current Opinion in Behavioral Sciences, 18, 83-90. https://doi.org/10.1016/j.cobeha.2017.07.018

Harari, G. M., Müller, S. R., Gosling, S. D., Harari, G. M., Müller, S. R., \& Gosling, S. D. (2018). Naturalistic assessment of situations using mobile sensing methods. In J. F. Rauthmann, R. A. Sherman, \& D. C. Funder (Eds.), The Oxford handbook of psychological situations. (pp. 299-311). https://doi.org/10.1093/oxfordhb/9780190263348.013.14

Harari, G. M., Vaid, S. S., Müller, S. R., Stachl, C., Marrero, Z., Schoedel, R., Bühner, M., \& Gosling, S. D. (2020). Personality sensing for theory development and assessment in the digital age. European fournal of Personality, 34(5), 649-669. https://doi.org/10.1002/per.2273

Horstmann, K. T., \& Ziegler, M. (2020). Assessing personality states: What to consider when constructing personality state measures. European fournal of Personality, 34(6), 1037-1059. https://doi.org/10.1002/per.2266

Israel, L. S. F., \& Schönbrodt, F. D. (2021). Predicting affective appraisals from facial expressions and physiology using machine learning. Behavior Research Methods, 53, 574-592. https://doi.org/10.3758/s13428-020-01435-y

Jach, H. K., Feuerriegel, D., \& Smillie, L. D. (2020). Decoding personality trait measures from resting EEG: An exploratory report. Cortex, 130, 158-171. https://doi.org/10.1016/j.cortex.2020.05.013

Jenkins, R., White, D., Van Montfort, X., \& Mike Burton, A. (2011). Variability in photos of the same face. Cognition, 121(3), 313-323. https://doi.org/10.1016/j.cognition.2011.08.001 
Jolly, E., \& Chang, L. J. (2019). The flatland fallacy: Moving beyond low-dimensional thinking. Topics in Cognitive Science, 11(2), 433-454. https://doi.org/10.1111/tops.12404

Judge, T. A., Simon, L. S., Hurst, C., \& Kelley, K. (2014). What I experienced yesterday is who I am today: Relationship of work motivations and behaviors to within-individual variation in the five-factor model of personality. The Journal of Applied Psychology, 99(2), 199-221. https://doi.org/10.1037/a0034485

Kachur, A., Osin, E., Davydov, D., Shutilov, K., \& Novokshonov, A. (2020). Assessing the Big Five personality traits using real-life static facial images. Scientific Reports, 10(1), Article 8487. https://doi.org/10.1038/s41598-020-65358-6

Kosinski, M. (2021). Facial recognition technology can expose political orientation from naturalistic facial images. Scientific Reports, 11, Article 100. https://doi.org/10.1038/s41598-020-79310-1

Kosinski, M., Stillwell, D., \& Graepel, T. (2013). Private traits and attributes are predictable from digital records of human behavior. Proceedings of the National Academy of Sciences of the United States of America, 110(15), 5802-5805. https://doi.org/10.1073/pnas.1218772110

Kulkarni, V., Kern, M. L., Stillwell, D., Kosinski, M., Matz, S., Ungar, L., Skiena, S., \& Schwartz, H. A. (2018). Latent human traits in the language of social media: An open-vocabulary approach. PLOS ONE, 13(11), Article e0201703. https://doi.org/10.1371/journal.pone.0201703

Kusner, M. J., \& Loftus, J. R. (2020). The long road to fairer algorithms. Nature, 578(7793), 34-36. https://doi.org/10.1038/d41586-020-00274-3

Lazer, D. M. J., Pentland, A., Watts, D. J., Aral, S., Athey, S., Contractor, N., Freelon, D., GonzalezBailon, S., King, G., Margetts, H., Nelson, A., Salganik, M. J., Strohmaier, M., Vespignani, A., \& Wagner, C. (2020). Computational social science: Obstacles and opportunities. Science, 369(6507), 1060-1062. https://doi.org/10.1126/science.aaz8170

Lu, J., Liu, A., Dong, F., Gu, F., Gama, J., \& Zhang, G. (2019). Learning under Concept Drift: A review. IEEE Transactions on Knowledge and Data Engineering, 31(2), 2346-2363. https://doi.org/10.1109/TKDE.2018.2876857

Matz, S. C., Kosinski, M., Nave, G., \& Stillwell, D. J. (2017). Psychological targeting as an effective approach to digital mass persuasion. Proceedings of the National Academy of Sciences of the United States of America, 114(48), 12714-12719. https://doi.org/10.1073/pnas.1710966114

Matz, S. C., \& Netzer, O. (2017). Using Big Data as a window into consumers' psychology. Current Opinion in Behavioral Sciences, 18, 7-12. https://doi.org/10.1016/j.cobeha.2017.05.009

Mehl, M. R., Pennebaker, J. W., Crow, D. M., Dabbs, J., \& Price, J. H. (2001). The Electronically Activated Recorder (EAR): A device for sampling naturalistic daily activities and conversations. Behavior Research Methods, Instruments, \& Computers, 33(4), 517-523. https://doi.org/10.3758/BF03195410

Miller, G. (2012). The smartphone psychology manifesto. Perspectives on Psychological Science, 7(3), 221-237. https://doi.org/10.1177/1745691612441215

Ortner, T. M., \& Schmitt, M. (2014). Advances and continuing challenges in objective personality testing. European fournal of Psychological Assessment, 30(3), 163-168. https://doi.org/10.1027/1015-5759/a000213 
Ozer, D. J., \& Benet-Martínez, V. (2006). Personality and the prediction of consequential outcomes. Annual Review of Psychology, 57(1), 401-421. https://doi.org/10.1146/annurev.psych.57.102904.190127

Park, G., Schwartz, H. A., Eichstaedt, J. C., Kern, M. L., Kosinski, M., Stillwell, D. J., Ungar, L. H., \& Seligman, M. E. P. (2015). Automatic personality assessment through social media language. fournal of Personality and Social Psychology, 108(6), 934-952. https://doi.org/10.1037/pspp0000020

Parry, D. A., Davidson, B. I., Sewall, C. J. R., Fisher, J. T., Mieczkowski, H., \& Quintana, D. S. (2021). A systematic review and meta-analysis of discrepancies between logged and self-reported digital media use. Nature Human Behaviour, 1-13. https://doi.org/10.1038/s41562-021-01117-5

Paulhus, D. L., \& Vazire, S. (2007). The self-report method. In R. W. Robins, R. C. Fraley, \& R. F. Krueger (Eds.), Handbook of research methods in personality psychology (pp. 224-239). Guilford Press.

Philpot, R., Liebst, L. S., Levine, M., Bernasco, W., \& Lindegaard, M. R. (2020). Would I be helped? Cross-National CCTV footage shows that intervention is the norm in public conflicts. The American Psychologist, 75(1), 66-75. https://doi.org/10.1037/amp0000469

Raento, M., Oulasvirta, A., \& Eagle, N. (2009). Smartphones. Sociological Methods \& Research, 37(3), 426-454. https://doi.org/10.1177/0049124108330005

Rauthmann, J. F., \& Sherman, R. A. (2018). The description of situations: Towards replicable domains of psychological situation characteristics. fournal of Personality and Social Psychology, 114(3), 482-488. https://doi.org/10.1037/pspp0000162

Rüegger, D., Stieger, M., Nißen, M., Allemand, M., Fleisch, E., \& Kowatsch, T. (2020). How are personality states associated with smartphone data? European fournal of Personality, 34(5), 687-713. https://doi.org/10.1002/per.2309

Schwartz, H. A., Eichstaedt, J. C., Kern, M. L., Dziurzynski, L., Ramones, S. M., Agrawal, M., Shah, A., Kosinski, M., Stillwell, D., Seligman, M. E. P., \& Ungar, L. H. (2013). Personality, gender, and age in the language of social media: The open-vocabulary approach. PLOS ONE, 8(9), Article e73791. https://doi.org/10.1371/journal.pone.0073791

Segalin, C., Celli, F., Polonio, L., Kosinski, M., Stillwell, D., Sebe, N., Cristani, M., \& Lepri, B. (2017). What your facebook profile picture reveals about your personality. $M M$ '17: Proceedings of the 25th ACM international conference on Multimedia, 460-468. https://doi.org/10.1145/3123266.3123331

Segalin, C., Perina, A., Cristani, M., \& Vinciarelli, A. (2017). The pictures we like are our image: Continuous mapping of favorite pictures into self-assessed and attributed personality traits. IEEE Transactions on Affective Computing, 8(2), 268-285. https://doi.org/10.1109/TAFFC.2016.2516994

Sen, I., Floeck, F., Weller, K., Weiss, B., \& Wagner, C. (2019). A total error framework for digital traces of humans. ArXiv. http://arxiv.org/abs/1907.08228 
Slavkovik, M., Stachl, C., Pitman, C., \& Askonas, J. (2021, May 19-21). Digital Voodoo dolls [Paper presentation]. AIES-2021: AAAI / ACM Conference on Artificial Intelligence, Ethics, and Society (Virtual conference).

Stachl, C., Au, Q., Schoedel, R., Gosling, S. D., Harari, G. M., Buschek, D., Völkel, S. T., Schuwerk, T., Oldemeier, M., Ullmann, T., Hussmann, H., Bischl, B., \& Bühner, M. (2020). Predicting personality from patterns of behavior collected with smartphones. Proceedings of the National Academy of Sciences of the United States of America, 117(30), 17680-17687. https://doi.org/10.1073/pnas.1920484117

Stachl, C., Pargent, F., Hilbert, S., Harari, G. M., Schoedel, R., Vaid, S., Gosling, S. D., \& Bühner, M. (2020). Personality research and assessment in the era of machine learning. European fournal of Personality, 34(5), 613-631. https://doi.org/10.1002/per.2257

Sterling, J., Jost, J. T., \& Bonneau, R. (2020). Political psycholinguistics: A comprehensive analysis of the language habits of liberal and conservative social media users. fournal of Personality and Social Psychology, 118(4), 805-834. https://doi.org/10.1037/pspp0000275

Stieger, M., Flückiger, C., Rüegger, D., Kowatsch, T., Roberts, B. W., \& Allemand, M. (2021). Changing personality traits with the help of a digital personality change intervention. Proceedings of the National Academy of Sciences of the United States of America, 118(8), Article e2017548118. https://doi.org/10.1073/pnas.2017548118

Stone, P. J., Dunphy, D. C., Smith, M. S., \& Ogilvie, D. M. (1966). The general inquirer: A computer approach to content analysis. M.I.T. Press.

Suen, H. Y., Hung, K. E., \& Lin, C. L. (2019). TensorFlow-Based automatic personality recognition used in asynchronous video interviews. IEEE Access: Practical Innovations, Open Solutions, 7, 61018-61023. https://doi.org/10.1109/ACCESS.2019.2902863

Tal, E. (2020). Measurement in science. In E. N. Zalta (Ed.), The stanford encyclopedia of philosophy. Metaphysics Research Lab, Stanford University.

Tausczik, Y. R., \& Pennebaker, J. W. (2010). The psychological meaning of words: LIWC and computerized text analysis methods. Journal of Language and Social Psychology, 29(1), 24-54. https://doi.org/10.1177/0261927X09351676

Tay, L., Woo, S. E., Hickman, L., \& Saef, R. M. (2020). Psychometric and validity issues in machine learning approaches to personality assessment: A focus on social media text mining. European Journal of Personality, 34(5), 826-844. https://doi.org/10.1002/per.2290

Todorov, A., Olivola, C. Y., Dotsch, R., \& Mende-Siedlecki, P. (2015). Social attributions from faces: Determinants, consequences, accuracy, and functional significance. Annual Review of Psychology, 66, 519-545. https://doi.org/10.1146/annurev-psych-113011-143831

Vazire, S., \& Mehl, M. R. (2008). Knowing me, knowing you: The accuracy and unique predictive validity of self-ratings and other-ratings of daily behavior. fournal of Personality and Social Psychology, 95(5), 1202-1216. https://doi.org/10.1037/a0013314

Voulodimos, A., Doulamis, N., Doulamis, A., \& Protopapadakis, E. (2018). Deep learning for computer vision: A brief review. Computational Intelligence and Neuroscience, 2018, Article 7068349. https://doi.org/10.1155/2018/7068349 
Yarkoni, T. (2012). Psychoinformatics: New horizons at the interface of the psychological and computing sciences. Current Directions in Psychological Science, 21(6), 391-397. https://doi.org/10.1177/0963721412457362

Youyou, W., Kosinski, M., \& Stillwell, D. (2015). Computer-based personality judgments are more accurate than those made by humans. Proceedings of the National Academy of Sciences of the United States of America, 112(4), 1036-1040. https://doi.org/10.1073/pnas.1418680112

\section{eapp}

Personality Science (PS) is an official journal of the European Association of Personality Psychology (EAPP).
(P) leibniz-psychology.org

PsychOpen GOLD is a publishing service by Leibniz Institute for Psychology (ZPID), Germany. 